\title{
NONLINEAR FUNCTIONAL RANDOM DIFFERENTIAL EQUATIONS IN BANACH ALGEBRAS
}

\author{
매III \\ B. C. DHAGE

\begin{abstract}
In this paper the existence as well as the existence of the extremal solutions for first order nonlinear perturbed functional random differential equations is proved under mixed Lipschitz, compactness and monotonic conditions.
\end{abstract}

\section{Statement of the problem}

The study of nonlinear differential and integral equations in a Banach algebra involving two nonlinearities in a multiplication form is initiated by Dhage [3] via fixed point methods and developed in a subsequent series of papers. See Dhage [4, 7] and the references therein. Thus several existence and uniqueness theorems have been proved for such random differential and integral equations under mixed Lipschitz and compactness type conditions in the course of time. Similarly, nonlinear random differential and integral equations in Banach algebras have also been studied in recent years by Dhage [6, 8] for various aspects of the solutions. But to the best of our knowledge nothing has been done so far for nonlinear functional random differential equations in Banach algebras. In this paper we shall discuss first order nonlinear functional random differential equations.

Let $\mathbb{R}$ denote the real line and let $I=[-r, 0]$ and $I_{0}=[0, a]$ be two closed and bounded intervals in $\mathbb{R}$ for some real numbers $r>0$ and $a>0$. Let $J=I_{0} \cup I$ and let $C\left(I_{0}, \mathbb{R}\right)$ denote the space of all continuous $\mathbb{R}$-valued functions on $I_{0}$. Define a supremum norm $\|\cdot\|_{C}$ in $C\left(I_{0}, \mathbb{R}\right)$ by

$$
\|x\|_{C}=\sup _{t \in I_{0}}|x(t)| .
$$

Clearly, $C$ is a Banach space which is also a Banach algebra with respect to this norm. For a given $t \in I$, define a continuous $\mathbb{R}$-valued function $x_{t}: I_{0} \rightarrow \mathbb{R}$ by

$$
x_{t}(\theta)=x(t+\theta), \quad \theta \in I_{0} .
$$

The function $x_{t}$ is called a history function and the space $C$ is called a history space of the dynamical systems in question.

Received May 17, 2005; revised November 8, 2005.

2000 Mathematics Subject Classification. 34F05, 34K05.

Key words and phrases. Random functional differential equation, existence theorem, extremal solutions, fixed points. 
Given a measurable space $(\Omega, \mathcal{A})$ and given a measurable function $\phi: \Omega \rightarrow C$, we study the functional random differential equation (in short FRDE)

$$
\left\{\begin{array}{lc}
\left(\frac{x(t)}{f(t, x(t, \omega), \omega)}\right)^{\prime}=g\left(t, x_{t}(\omega), \omega\right) & \text { a.e. } t \in I, \\
x_{0}(\omega)=\phi(\omega), & t \in I_{0},
\end{array}\right.
$$

for all $\omega \in \Omega$, where $f: J \times \mathbb{R} \times \Omega \rightarrow \mathbb{R} \backslash\{0\}$ is continuous and $g: J \times C \times \Omega \rightarrow \mathbb{R}$.

Definition 1.1. By a random solution of the FRDE (1.1) we mean a measurable function $x: \Omega \rightarrow C(J, \mathbb{R}) \cap A C(I, \mathbb{R})$ that satisfies the equations in (1.1) on $J$, where $A C(I, \mathbb{R})$ is the space of all absolutely continuous real-valued functions on $I$.

Special cases of the FRDE (1.1) have already been discussed in the literature. For example, when $f(t, x, \omega)=1$ for all $t \in I, \omega \in \Omega$ and $x \in \mathbb{R}, 1$ being the multiplicative unity in $\mathbb{R}$, the FRDE (1.1) reduces to

$$
\begin{cases}x^{\prime}(t)=g\left(t, x_{t}(\omega), \omega\right) & \text { a.e. } t \in I, \\ x_{0}(\omega)=\phi(\omega), & t \in I_{0},\end{cases}
$$

for all $\omega \in \Omega$.

The FRDE (1.2) has been studied in [8] for different aspects of the random solutions. Further, if the random parameter $\omega$ is absent, then FRDE (1.1) reduces to the deterministic functional differential equation (FDE),

$$
\left\{\begin{array}{l}
\left(\frac{x(t)}{f(t, x(t))}\right)^{\prime}=g\left(t, x_{t}\right) \quad \text { a.e. } t \in I, \\
x(t)=\phi(t), \\
\quad t \in I_{0}
\end{array}\right.
$$

The FDE (1.3) has been discussed in Dhage et al. 11] for existence as well as for the existence of extremal solutions under the mixed compactness and monotonic conditions on the nonlinearities involved in it.

In this paper we shall study the existence as well as existence of extremal solutions to FRDE (1.1) under Lipschitz, compactness and monotonic conditions. Our results include the existence theorems for FRDE (1.2) and FDE (1.3) as special cases.

\subsection{Random fixed point theory}

Let $(\Omega, \mathcal{A})$ be a measurable space and let $X$ be a separable Banach space. By $\beta_{X}$ we denote the $\sigma$-algebra of all Borel subsets of $X$. A mapping $x: \Omega \rightarrow X$ is called measurable if for any Borel subset $B$ of $X$,

$$
x^{-1}(B)=\{\omega \in \Omega \mid x(\omega) \in B\} .
$$


A mapping $T: \Omega \times X \rightarrow X$ is called a random operator if $\omega \rightarrow T(\omega, x)$ is measurable for all $x \in X$ and we denote it by $T(\omega) x=T(\omega, x)$.

Let $T: X \rightarrow X$. Then $T$ is called continuous if for any open set $U$ in $X, T^{-1}(U)$ is open in $X . T$ is called compact if $T(X)$ is relatively compact subset of $X . T$ is called totally bounded if for any bounded subset $S$ of $X, T(S)$ is a totally bounded subset of $X$. Finally, $T$ is called completely continuous if it is continuous and totally bounded on $X$. Note that every compact operator is totally bounded, but the converse may not be true. However these two notions are equivalent on a bounded subset of $X$. Similarly, a random operator $T: \Omega \times X \rightarrow X$ is called continuous (a compact, totally bounded and completely continuous) if the operator $T(\omega)$ is continuous (a compact, totally bounded and completely continuous) for each $\omega \in \Omega$. A measurable mapping $\xi: \Omega \rightarrow X$ is called a random fixed point of the random operator $T: \Omega \times X \rightarrow X$ if $T(\omega) \xi(\omega)=\xi(\omega)$ for all $\omega \in \Omega$.

Definition 2.1. A random operator $T: \Omega \times X \rightarrow X$ is called $\mathcal{D}$-Lipschitz if there exists a continuous and nondecreasing function $\phi_{\omega}: \Omega \times \mathbb{R}^{+} \rightarrow \mathbb{R}^{+}$satisfying for each $\omega \in \Omega$,

$$
\|T(\omega) x-T(\omega) y\|=\phi_{\omega}(\|x-y\|)
$$

for all $x, y \in X$, where $\phi_{\omega}(r)=\phi(\omega, r)$ with $\phi(\omega, 0)=0, \forall \omega \in \Omega$. The function $\phi$ is called a $D$-function of the random operator $T(\omega)$ on $X$. In the special case when $\phi_{\omega}(r)=a(\omega) r$ for some measurable function $a: \Omega \rightarrow \mathbb{R}^{+} \backslash\{0\}$, the random operator $T(\omega)$ is called Lipschitz with the Lipschitz constant $a(\omega)$. In particular, if $a(\omega)<1$ for each $\omega \in \Omega$, $T(\omega)$ is called a contraction on $X$. Finally, if $a(\omega)=\ell<1, \forall \omega \in \Omega$, then the random operator $T(\omega)$ is called a uniform contraction on $X$.

We use the following essential fixed point theorem of Dhage 7] for establishing the main existence results of this paper.

Theorem 2.1. Let $X$ be a separable Banach algebra and let $(\Omega, \mathcal{A})$ be a measurable space. Let $A, B: \Omega \times X \rightarrow X$ be two random operators satisfying for each $\omega \in \Omega$,

(a) $A(\omega)$ is $\mathcal{D}$-Lipschitz with a $\mathcal{D}$-function $\phi$,

(b) $B(\omega)$ is continuous and compact, and

(c) the set $\mathcal{E}=\{x \in X \mid A(\omega) x B(\omega) x=\lambda(\omega) x\}$ is bounded for any measurable function $\lambda: \Omega \rightarrow \mathbb{R}$ with $\lambda(\omega)>1, \forall \omega \in \Omega$.

Then the random equation

$$
A(\omega) x B(\omega) x=x
$$

has a random solution whenever $M(\omega) \phi_{\omega}(\rho)<\rho, \rho>0$ for each $\omega \in \Omega$, where

$$
M(\omega)=\|B(\omega)(X)\|=\sup \{\|x(\omega)\|: x \in B(\omega)(X)\} .
$$

Proof. The proof appears in Dhage 7] and involves the use of measures of noncompactness and condensing mappings in Banach spaces. Here we give the proof by using the elementary ideas from the linear functional analysis. 
Let $\omega \in \Omega$ be fixed. Then, by a fixed point theorem of Dhage 7], the operator equation $A(\omega) x B(\omega) x=x$ has a solution in $X$. Define a multi-valued map $F: \Omega \rightarrow X$ by

$$
F(\omega)=\{x \in X \mid A(\omega) x B(\omega) x=x\} .
$$

Clearly, $F(\omega)$ is non-empty for each $\omega \in \Omega$. Further, it is easy to see that $F(\omega)$ is compact for each $\omega \in \Omega$. See Dhage 7] and the references therein. We shall show that the multi-valued map $\mathrm{F}$ is measurable on $\Omega$. Now, for any closed subset $C$ of $X$, denote

$$
L(C)=\bigcap_{n=1}^{\infty} \bigcup_{x_{i} \in C_{n}}\left\{\omega \in \Omega \mid\left\|x_{i}-A(\omega) x_{i} B(\omega) x_{i}\right\|<\frac{2}{n}\right\}
$$

where $C_{n}=\left\{x \in X \mid d(x, C)<\frac{1}{n}\right\}$ and $d(x, C)=\inf \{d(x, c) \mid c \in C\}$.

Obviously, $L(C)$ is measurable. Now proceeding with the arguments similar to that in the proof of Theorem 2.1, of Itoh 14], it is proved that $F^{-1}(C)=L(C)$. Hence $F$ is measurable on $\Omega$. Since $F(\omega)$ is compact, it has closed values for each $\omega \in \Omega$. Now an application of a selection theorem of Kuratowskii and Ryll-Nardzewskii $[16]$ yields that $F$ has a measurable selector $\xi$ on $\Omega$. This further implies that $\xi(\omega)=A(\omega) \xi(\omega) B(\omega) \xi(\omega)$ for each $\omega \in \Omega$. This completes the proof.

Corollary 2.2. Let $(\Omega, \mathcal{A})$ be a measurable algebra and let $X$ be a separable Banach space. Let $A, B: \Omega \times X \rightarrow X$ be two random operators satisfying for each $\omega \in \Omega$,

(a) $A(\omega)$ is Lipschitz with a $\mathcal{D}$ - Lipschitz constant $a(\omega)$.

(b) $B(\omega)$ is compact and continuous, and

(c) the set $\mathcal{E}=\{x: \Omega \rightarrow X \mid A(\omega) x B(\omega) x=\lambda x\}$ is bounded for any real number $\lambda>1$.

Then the random equation (1.1) has a random solution, whenever $a(\omega) M(\omega)<1$ for each $\omega \in \Omega$, where

$$
M(\omega)=\|B(\omega)(X)\|=\sup \{\|x(\omega)\|: x \in B(\omega)(X)\}
$$

Proof. Since every Lipschitz mapping is $\mathcal{D}$-Lipschitz and every constant real-function on $\Omega$ is measurable, the desired result follows by a direct application of Theorem 2.1. This completes the proof.

In the following section we apply the abstract results of this section for proving the existence of a random solution to FRDE (1.1) under suitable conditions.

\section{Existence theory}

If a function $t \mapsto x(t, \omega)$ is continuous for each $\omega \in \Omega$, then in this case we write $x: \Omega \rightarrow C(J, \mathbb{R})$. Now the FRDE (1.1) is equivalent to the random integral equation (in 
short RIE),

$$
\begin{aligned}
& x(t, \omega)=[f(t, x(t, \omega), \omega)]\left(\phi(0, \omega)+\int_{0}^{t} g\left(s, x_{s}(\omega), \omega\right) d s\right), \quad t \in I, \\
& x(t, \omega)=\phi(t, \omega), \quad t \in I_{0},
\end{aligned}
$$

for each $\omega \in \Omega$, assuming that $f(0, \phi(0, \omega), \omega)=1$. (See (f3) below). We shall seek the solution of RIE (3.1)-(3.2) in the space $C(J, \mathbb{R})$ under suitable conditions on the functions $f$ and $g$.

We need the following definition in the sequel.

Definition 3.1. A mapping $\beta: I \times C \times \Omega \rightarrow \mathbb{R}$ is said to satisfy a condition of $L(\omega)$-Carathéodory if for each $\omega \in \Omega$,

(i) $t \mapsto \beta(t, x, \omega)$ is measurable for each $x \in C$, and

(ii) $x \mapsto \beta(t, x, \omega)$ is continuous almost everywhere for $t \in I$.

Further, a $L(\omega)$-Carathéodory function $\beta$ is called $L_{C}^{1}-\omega$-Carathéodory if

(iii) there exists a function $h: \Omega \rightarrow L^{1}(I, \mathbb{R})$ such that

$$
|\beta(t, x, \omega)| \leq h(t, \omega) \text { a.e. } t \in I,
$$

for all $\omega \in \Omega$ and for all $x \in C$.

We consider the following hypotheses in the sequel.

(f1) The function $\omega \rightarrow f(t, x, \omega)$ is measurable for all $t \in I$ and $x \in \mathbb{R}$.

(f2) The function $f: I \times \mathbb{R} \times \Omega \rightarrow \mathbb{R} \backslash\{0\}$ is continuous and there exists a function $\alpha \in B(I, \mathbb{R})$ with bound $\|\alpha(\omega)\|$ satisfying for each $\omega \in \Omega$,

$$
|f(t, x, \omega)-f(t, y, w)| \leq \alpha(t, \omega)\|x(\omega)-y(\omega)\|, \quad \text { a.e. } t \in I,
$$

for all $x, y \in \mathbb{R}$.

(f3) $f(0, \phi(0, \omega), \omega)=1, \forall \omega \in \Omega$.

(g1) The function $(t, \omega) \rightarrow g(t, x, \omega)$ is measurable for all $t \in I$ and $x \in C$.

(g2) The function $g$ is $L_{X}^{1}-\omega$-Carathéodory.

(g3) There exists a function $\gamma: \Omega \rightarrow L^{1}(I, \mathbb{R})$ with $\gamma(t, \omega)>0$ a.e. $t \in I$ and a continuous nondecreasing function $\psi:[0, \infty) \rightarrow(0, \infty)$ satisfying for each $\omega \in \Omega$,

$$
|g(t, x, \omega)| \leq \gamma(t, \omega) \psi\left(\|x(\omega)\|_{C}\right) \text { a.e. } t \in I,
$$

for all $x \in C$.

Theorem 3.1. Assume that the hypotheses (f1)-(f3) and (g1)-(g3) hold. Suppose further that

$$
\int_{c}^{\infty} \frac{d s}{\psi(s)}>\frac{F(\omega)}{1-C_{1}(\omega)}\|\gamma(\omega)\|_{L^{1}}
$$


where

$$
\begin{gathered}
C_{1}(\omega)=\|\alpha(\omega)\|\left(\|\phi(\omega)\|_{C}+\|h(\omega)\|_{L^{1}}\right)<1, \\
C_{2}(\omega)=F(\omega)\left|\|\phi(\omega)\|_{C}, \quad F(\omega)=\sup _{t \in I}\right| f(t, 0, \omega) \mid, \quad c=\frac{C_{2}(\omega)}{1-C_{1}(\omega)} .
\end{gathered}
$$

Then the FRDE (1.1) has a random solution on $J$.

Proof. Let $X=C(J, \mathbb{R})$ and define two mappings $A, B: \Omega \times X \rightarrow X$, by

$$
A(\omega) x(t)= \begin{cases}f(t, x(t, \omega), \omega), & \text { a.e. } t \in I, \\ 1, & t \in I_{0},\end{cases}
$$

and

$$
B(\omega) x(t)= \begin{cases}\phi(0, \omega)+\int_{0}^{t} g\left(t, x_{s}(\omega), \omega\right), & \text { a.e. } t \in I, \\ \phi(t, \omega), & t \in I_{0} .\end{cases}
$$

Then the FRIE (3.1) is transformed into the random operator equation

$$
A(\omega) x(t) B(\omega) x(t)=x(t, \omega)
$$

for $t \in J$ and $\omega \in \Omega$.

We shall show that the operators $A(\omega)$ and $B(\omega)$ satisfy all the conditions of Corollary 2.2. on $X$. This will be done in the following steps.

Step I : First we show that $A(\omega)$ and $B(\omega)$ are random operators on $X$. Since the function $f(t, x, \omega)$ is measurable in $\omega$ for all $t \in I$ and $x \in \mathbb{R}$, and since the constant function is measurable on $\Omega$, the function $\omega \rightarrow A(\omega) x$ is measurable for all $x \in X$. Hence $A(\omega)$ is a random operator on $X$. Now by (H3), the function $(t, x, \omega) \rightarrow g(t, x, \omega)$ is measurable for all $t \in I$ and $x \in C$. We know that the Riemann integral, as a limit of a finite sum of measurable functions, is again measurable. Therefore, the function $\omega \rightarrow \int_{0}^{t} g\left(s, x_{s}(\omega), \omega\right) d s$ is measurable. Hence $B(\omega)$ is a random operator on $X$.

Again, since the function

$$
t \mapsto A(\omega) x(t)= \begin{cases}f(t, x(t, \omega), \omega), & \text { a.e. } t \in I, \\ 0, & t \in I_{0},\end{cases}
$$

and

$$
t \mapsto B(\omega) x(t)=\left\{\begin{array}{lc}
\phi(0, \omega)+\int_{0}^{t} g\left(s, x_{s}(\omega), \omega\right) d s, & \text { a.e. } t \in I, \\
\phi(t, \omega), & t \in I_{0},
\end{array}\right.
$$

are continuous, the functions $A(\omega) x(\cdot)$ and $B(\omega) x(\cdot)$ are continuous and hence bounded and measurable on $J$ for each $\omega \in \Omega$. Hence $A(\omega)$ and $B(\omega)$ define the random operators $A, B: \Omega \times X \rightarrow X$. 
Step II : Next we show that $A(\omega)$ is a Lipschitz random operator on $X$. Let $x, y \in X$. Then by (H1),

$$
\begin{aligned}
|A(\omega) x(t)-A(\omega) y(t)| & =|f(t, x(t, \omega), \omega)-f(t, y(t, \omega), \omega)| \\
& \leq \alpha(t, \omega)|x(t, \omega)-y(t, \omega)| \\
& \leq\|\alpha(\omega)\|\|x(\omega)-y(\omega)\|,
\end{aligned}
$$

for all $t \in I$. Similarly,

$$
\begin{aligned}
|A(\omega) x(t)-A(\omega) y(t)| & =|\phi(t, \omega)-\phi(t, \omega)| \\
& \leq\|\alpha(t, \omega)\|\|x(\omega)-y(\omega)\|,
\end{aligned}
$$

for all $t \in I_{0}$. Thus

$$
|A(\omega) x(t)-A(\omega) y(t)| \leq\|\alpha(\omega)\|\|x(\omega)-y(\omega)\|
$$

for all $t \in J$ and $\omega \in \Omega$. Taking the supremum over $t$ in the above inequality, we obtain

$$
\|A(\omega) x-A(\omega) y\| \leq\|\alpha(\omega)\|\|x(\omega)-y(\omega)\| .
$$

This shows that $A(\omega)$ is a Lipschitz random operator on $X$ with the Lipschitz constant $\|\alpha(\omega)\|$, for all $\omega \in \Omega$.

Step III : Next we show that $B(\omega)$ is a continuous and compact random operator on $X$. Using the standard arguments as in Dhage [8], it is shown that $B(\omega)$ is a continuous random operator on $X$. To show that $B(\omega)$ is compact, it suffices to show that $B(\omega)(X)$ is a uniformly bounded and equi-continuous set in $X$ for each $\omega \in \Omega$. First we show that $B(\omega)(X)$ is uniformly bounded for each $\omega \in \Omega$. Let $x \in X$ be arbitrary. Thus

$$
B(\omega) x(t)= \begin{cases}\phi(0, \omega)+\int_{0}^{t} g\left(s, x_{s}(\omega), \omega\right) d s, & \text { a.e. } t \in I, \\ \phi(t, \omega), & t \in I_{0} .\end{cases}
$$

Since $g$ is $L_{X}^{1}(\omega)$-Carathéodory, we have

$$
\begin{aligned}
|B(\omega) x(t)| & \leq\|\phi(\omega)\|_{C}+\int_{0}^{t}\left|g\left(s, x_{s}(\omega), \omega\right)\right| d s \\
& \leq\|\phi(\omega)\|_{C}+\int_{0}^{t} h(s, \omega) d s \\
& \leq\|\phi(\omega)\|_{C}+\int_{0}^{a} h(s, \omega) d s \\
& \leq\|\phi(\omega)\|_{C}+\|h(\omega)\|_{L^{1}} .
\end{aligned}
$$

Taking the supremum over $t$, one obtains

$$
\|B(\omega) x\| \leq K,
$$


for all $x \in X$, where $K=\|\phi(\omega)\|_{C}+\|h(\omega)\|_{L^{1}}$.

This shows that $B(\omega)(X)$ is a uniformly bounded subset of $X$ for each $\omega \in \Omega$.

Secondly, we show that $B(\omega)(X)$ is an equi-continuous set in $X$ for each $\omega \in \Omega$. There are three cases:

Case I : Let $t, \tau \in I$. Then, for any $x \in X$, we have by (3.5)

$$
\begin{aligned}
|B(\omega) x(t)-B(\omega) x(\tau)| & \leq\left|\int_{0}^{t} g\left(s, x_{s}(\omega), \omega\right) d s-\int_{0}^{\tau} g\left(s, x_{s}(\omega), \omega\right) d s\right| \\
& \leq\left|\int_{\tau}^{t}\right| g\left(s, x_{s}(\omega), \omega\right)|d s| \\
& \leq\left|\int_{\tau}^{t} h(s, \omega) d s\right| \\
& \leq|p(t, \omega)-p(\tau, \omega)|
\end{aligned}
$$

where $p(t, \omega)=\int_{0}^{t} h(s, \omega) d s$. Now $p$ is a continuous function on the compact interval $I$, so it is uniformly continuous and hence

$$
|B(\omega) x(t)-B(\omega) x(\tau)| \rightarrow 0 \quad \text { as } t \rightarrow \tau \text { for each } \omega \in \Omega .
$$

Case II : Again let $\tau \in I_{0}$ and $t \in I$. Then, we have

$$
\begin{aligned}
|B(\omega) x(t)-B(\omega) x(\tau)| & \leq|\phi(0, \omega)-\phi(\tau, \omega)|+\int_{0}^{t}\left|g\left(s, x_{s}(\omega), \omega\right)\right| d s \\
& \leq|\phi(0, \omega)-\phi(\tau, \omega)|+|p(t, \omega)-p(0, \omega)|
\end{aligned}
$$

where the function $p$ defined above. Again $\phi$ is continuous on the compact interval $I_{0}$ and the function $p$ is continuous on the compact interval $I$, so they are uniformly continuous there, and hence

$$
|B(\omega) x(t)-B(\omega) x(\tau)| \rightarrow 0 \quad \text { as } t \rightarrow \tau .
$$

Case III: Similarly, let $t, \tau \in I_{0}$. Thus we have

$$
|B(\omega) x(t)-B(\omega) x(\tau)|=|\phi(t, \omega)-\phi(t, \omega)| \rightarrow 0 \quad \text { as } t \rightarrow \tau .
$$

Thus, in all three cases, we have

$$
|B(\omega) x(t)-B(\omega) x(\tau)| \rightarrow 0 \quad \text { as } t \rightarrow \tau,
$$

for all $t, \tau \in J$ and $\omega \in \Omega$. Hence $B(\omega)(X)$ is an equi-continuous set in $X$ for each $\omega \in \Omega$. This, in view of the Arzelá-Ascolli theorem, further implies that $B(\omega)(X)$ is compact for each $\omega \in \Omega$. Hence $B(\omega)$ is a continuous and compact random operator on $X$. 
Step IV : Here, we show that $\alpha(\omega) M(\omega)<1$. We have

$$
\begin{aligned}
M(\omega) & =\|B(\omega)(X)\| \\
& =\sup \{\|B(\omega) x\|: x \in X\} \\
& =\sup _{x \in X}\left\{\max _{t \in J}|B(\omega) x(t)|\right\} \\
& \leq\|\phi(\omega)\|_{C}+\sup _{x \in X}\left\{\max _{t \in J}\left|\int_{0}^{t}\right| g\left(s, x_{s}(\omega), \omega\right) \| d s\right\} \\
& \leq\|\phi(\omega)\|_{C}+\max _{t \in I} \int_{0}^{t} h(s, \omega) d s \\
& =\|\phi(\omega)\|_{C}+\int_{0}^{a} h(s, \omega) d s \\
& =\|\phi(\omega)\|_{C}+\|h(\omega)\|_{L^{1}} .
\end{aligned}
$$

Therefore $\alpha(\omega) M(\omega)=\|\alpha(\omega)\|\left(\|\phi(\omega)\|_{C}+\|h(\omega)\|_{L^{1}}\right)$ and

$$
\alpha(\omega) M(\omega)=\|\alpha(\omega)\|\left(\|\phi(\omega)\|_{C}+\|h(\omega)\|_{L^{1}}\right)<1
$$

for all $\omega \in \Omega$, by our assumption.

Step V : Finally, we show that the condition (c) of Corollary 2.2 is satisfied. Let $u \in \mathcal{E}$ be arbitrary. Then we have for all $\omega \in \Omega$,

$$
\begin{aligned}
\lambda u(t, \omega) & =A(\omega) u(t) B(\omega) u(t) \\
& = \begin{cases}{[f(t, u(t, \omega), \omega)]\left(\phi(0, \omega)+\int_{0}^{t} g\left(s, u_{s}(\omega), \omega\right) d s\right),} & \text { a.e. } t \in I, \\
\phi(t, \omega), & t \in I_{0},\end{cases}
\end{aligned}
$$

for some real number $\lambda>1$.

If $t \in I_{0}$, then we have

$$
|u(t, \omega)|=\lambda^{-1}|\phi(t, 0)| \leq\|\phi(\omega)\|_{C}<\infty
$$


or all $\omega \in \omega$. Therefore, if $t \in I$, we have

$$
\begin{aligned}
|u(t, \omega)| \leq & \lambda^{-1}[|f(t, u(t, \omega), \omega)|]\left(|\phi(0, \omega)|+\int_{0}^{t}\left|g\left(s, u_{s}(\omega), \omega\right)\right| d s\right) \\
\leq & {[|f(t, u(t, \omega), \omega)-f(t, 0, \omega)+f(t, 0, \omega)|] \times } \\
& \times\left(\|\phi(\omega)\|_{C}+\int_{0}^{t}\left|g\left(s, u_{s}(\omega), \omega\right)\right| d s\right) \\
\leq & {[a(t, \omega) \| u \omega) \|+|f(t, 0, \omega)|] \times } \\
& \times\left(\|\phi(\omega)\|_{C}+\int_{0}^{t}\left|g\left(s, u_{s}(\omega), \omega\right)\right| d s\right) \\
\leq & a(\omega)\|u(\omega)\|\left(\|\phi(\omega)\|_{C}+\int_{0}^{t}\left|g\left(s, u_{s}(\omega), \omega\right)\right| d s\right) \\
& +|f(t, 0, \omega)|\left(\|\phi(\omega)\|_{C}+\int_{0}^{t} \gamma(s, \omega) \psi\left(\left\|u_{s}(\omega)\right\|_{C}\right) d s\right) \\
\leq & a(\omega)\|u(\omega)\|\left(\|\phi(\omega)\|_{C}+\|h(\omega)\|_{L^{1}}\right) \\
& +\sup _{t \in I}|f(t, 0, \omega)|\|\phi(\omega)\|_{C} \\
& +\sup _{t \in I}|f(t, 0, \omega)| \int_{0}^{t} \gamma(s, \omega) \psi\left(\left\|u_{s}(\omega)\right\|_{C}\right) d s \\
\leq & C_{1}(\omega)\left\|u_{t}(\omega)\right\|_{C}+C_{2}(\omega)+F(\omega) \int_{0}^{t} \gamma(s, \omega) \psi\left(\left\|u_{s}(\omega)\right\|_{C}\right) d s
\end{aligned}
$$

where

$$
\begin{gathered}
C_{1}(\omega)=\|\alpha(\omega)\|\left(\|\phi(\omega)\|_{C}+\|h(\omega)\|_{L^{1}}\right) \\
C_{2}(\omega)=F(\omega)\left|\|\phi(\omega)\|_{C}, \quad F(\omega)=\sup _{t \in I}\right| f(t, 0, \omega) \mid .
\end{gathered}
$$

Let $m(t, \omega)=\sup _{s \in[-r, t]}|u(t, \omega)|$. Then one has $|u(t, \omega)| \leq m(t, \omega)$ and $\left\|u_{t}(\omega)\right\|_{C} \leq$ $m(t, \omega)$ for all $t \in I$ and $\omega \in \Omega$. Then there is a $t^{*} \in[-r, t]$ such that

$$
m(t, \omega)=\left|u\left(t^{*}, \omega\right)\right|, \quad \forall \omega \in \Omega
$$

Hence from the previous inequality, it follows that

$$
\begin{aligned}
m(t, \omega) & =\left|u\left(t^{*}, \omega\right)\right| \\
& \leq C_{1}(\omega)\left\|u_{t^{*}}(\omega)\right\|_{C}+C_{2}(\omega)+F(\omega) \int_{0}^{t^{*}} \gamma(s, \omega) \psi\left(\left\|u_{s}(\omega)\right\|_{C}\right) d s \\
& \leq C_{1}(\omega) m(t, \omega)+C_{2}(\omega)+F(\omega) \int_{0}^{t} \gamma(s, \omega) \psi(m(s, \omega)) d s,
\end{aligned}
$$

or

$$
m(t, \omega) \leq \frac{C_{2}(\omega)}{1-C_{1}(\omega)}+\frac{F(\omega)}{1-C_{1}(\omega)} \int_{0}^{t} \gamma(s, \omega) \psi(m(s, \omega)) d s:=w(t, \omega)
$$


Differentiating w.r.t $t$ gives

$$
\begin{gathered}
w^{\prime}(t, \omega)=\frac{F(\omega)}{1-C_{1}(\omega)} \gamma(t, \omega) \psi(m(t, \omega)), \\
w(0, \omega)=\frac{C_{2}(\omega)}{1-C_{1}(\omega)},
\end{gathered}
$$

which further implies that

$$
\begin{gathered}
w^{\prime}(t, \omega) \leq \frac{F(\omega)}{1-C_{1}(\omega)} \gamma(t, \omega) w(w(t, \omega)) \\
w(0, \omega)=\frac{C_{2}(\omega)}{1-C_{1}(\omega)}
\end{gathered}
$$

or

$$
\frac{w(t, \omega)}{\psi(w(t, \omega))} \leq \frac{F(\omega)}{1-C_{1}(\omega)} \gamma(t, \omega), \quad w(0, \omega)=\frac{C_{2}(\omega)}{1-C_{1}(\omega)}
$$

Integrating from 0 to $t$ yields that

$$
\int_{0}^{t} \frac{w(s, \omega)}{\psi(w(s, \omega))} d s \leq \frac{F(\omega)}{1-C_{1}(\omega)} \int_{0}^{t} \gamma(s, \omega) d s .
$$

By change of the variable formula, we get

$$
\begin{aligned}
\int_{w(0, \omega)}^{w(t, \omega)} \frac{d s}{\psi(s)} & \leq \frac{F(\omega)}{1-C_{1}(\omega)} \int_{0}^{t} \gamma(s, \omega) d s \\
& \leq \frac{F(\omega)}{1-C_{1}(\omega)} \int_{0}^{a} \gamma(s, \omega) d s \\
& \leq \frac{F(\omega)}{1-C_{1}(\omega)}\|\gamma(\omega)\|_{L^{1}} \\
& <\int_{c}^{\infty} \frac{d s}{\psi(s)}, \quad c=\frac{C_{2}(\omega)}{1-C_{1}(\omega)}
\end{aligned}
$$

Now, by an application of mean value theorem, it follows that there is a constant $M>0$ such that

$$
w(t, \omega) \leq M, \quad \forall t \in I \text { and } \omega \in \Omega
$$

This further implies that

$$
|u(t, \omega)| \leq M, \quad \forall t \in J \text { and } \omega \in \Omega
$$

Hence the set $\mathcal{E}$ is bounded and the condition (c) of Corollary 2.2 follows. Hence the random operator equation (3.6), and consequently the FRDE (1.1), has a random solution on $J$. This completes the proof. 


\section{Existence of extremal random solutions}

\subsection{Monotone random operators}

A non-empty closed subset $K$ of a separable Banach algebra $X$ is called a cone if (a) $K+K \subseteq K$,

(b) $\lambda K \subseteq K$ for all $\lambda \in \mathbb{R}, \lambda \geq 0$, and

(c) $\{-K\} \cap K=0$, where 0 is a zero element of $X$.

Again, the cone $K$ in $X$ is called positive if

(d) $K \circ K \subseteq K$, where " $\circ "$ is a multiplication composition in $X$.

We define an order relation $\leq$ in $X$ with the help of a cone $K$ as follows. Let $x, y \in X$. Then

$$
x \leq y \quad \text { iff } y-x \in K
$$

The Banach algebra $X$ together with the order relation $\leq$ is called an ordered Banach algebra and it is usually denoted by $(X,\|\cdot\|, \leq)$ or simply by $(X, K)$. A cone $K$ in $X$ is called normal if the norm $\|\cdot\|$ is semi-monotone on $K$, i.e. if $x, y \in K$ with $x \leq y$, then there exists a constant $N>0$ such that $\|x\| \leq N\|y\|$. Let $u, v \in X$ be such that $u \leq v$. Then the order interval $[u, v]$ is a set in $X$ defined by

$$
[u, v]=\{x \in X \mid u \leq x \leq v\} .
$$

It is known that if the cone $K$ in $X$ is normal, then every order interval is norm-bounded set in $X$. Let $a, b: \Omega \rightarrow X$ be two measurable functions. Then by $a \leq b$ we mean $a(\omega) \leq b(\omega)$ for all $\omega \in \Omega$. In this case, the random order interval $[a, b]$ is defined to be a set in $X$ given by

$$
[a, b]=\{x \in X \mid a(\omega) \leq x \leq b(\omega), \forall \omega \in \Omega\}=\bigcap_{\omega \in \Omega}[a(\omega), b(\omega)]
$$

Definition 4.1. A random operator $T: \Omega \times X \rightarrow X$ is called monotone nondecreasing if for any $x, y \in X, x \leq y$ implies $T(\omega) x \leq T(\omega) y$ for all $\omega \in \Omega$. Again, the random operator $T: \Omega \times X \rightarrow X$ is called positive if $T(\omega)(X) \subset K$ for each $\omega \in \Omega$.

We use the following fixed point theorem of Dhage [3] in the sequel.

Theorem 4.1. Let $A, B: \Omega \times X \rightarrow X$ be two positive and monotone nondecreasing random operators satisfying for each $\omega \in \Omega$,

(a) $A(\omega)$ is Lipschitz with the Lipschitz constant $\alpha(\omega)$,

(b) $B(\omega)$ is completely continuous, and

(c) there exists two measurable functions $a, b: \Omega \times X \rightarrow X$ such that $a(\omega) \leq A(\omega) a B(\omega) a$ and $A(\omega) b B(\omega) b \leq b(\omega)$.

Further, if the cone $K$ in $X$ is positive and normal, then the random equation

$$
A(\omega) x B(\omega) x=x
$$


has the least random solution $\xi_{*}$ and the greatest random solution $\xi^{*}$ in $[a, b]$ whenever $a(\omega) M(\omega)<1$ for each $\omega \in \Omega$, where $M(\omega)=\|B(\omega)([a, b])\|$.

Moreover,

$$
\xi_{*}(\omega)=\lim x_{n}(\omega) \text { and } \xi_{*}(\omega)=\lim y_{n}(\omega)
$$

where,

$$
\begin{aligned}
& x_{n+1}(\omega)=A(\omega) x_{n}+B(\omega) x_{n}, \quad n \geq 0 \text { with } x_{0}(\omega)=a(\omega), \\
& y_{n+1}(\omega)=A(\omega) y_{n}+B(\omega) y_{n}, \quad n \geq 0 \text { with } y_{0}(\omega)=a(\omega) .
\end{aligned}
$$

\subsection{Extremal random solutions}

First we shall prove the existence of extremal random solutions to the FRDE (1.1) in the space $C(J, \mathbb{R})$ of all continuous real-valued functions on $J$, and then, as applications, we shall obtain the existence theorems for extremal random solutions to the FRDE (1.1) on $J$.

Define an order relation $\leq$ in $C(J, \mathbb{R})$ by a cone $K$ in $C(J, \mathbb{R})$ defined by

$$
K=\{x \in C(J, \mathbb{R}) \mid x \geq 0\} .
$$

Clearly, a cone $K$ is positive and normal in $C(J, \mathbb{R})$.

Definition 4.2. A random solution $\xi_{M}$ to the FRDE (1.1) is said to be maximal if for any other random solution $x$, we have $x(t, \omega) \leq \xi_{M}(t, \omega)$ for all $t \in J$ and $\omega \in \Omega$. Similarly, a minimal random solution to the FRDE (1.1) is defined.

We need the following definitions in the sequel.

Definition 4.3. A mapping $\beta: I \times C \times \Omega \rightarrow \mathbb{R}$ is said to satisfy a condition of $L(\omega)$-Chandrabhan if for each $\omega \in \Omega$,

(i) $t \mapsto \beta(t, x, \omega)$ is measurable for each $x \in C$, and

(ii) $x \mapsto \beta(t, x, \omega)$ is nondecreasing almost everywhere for $t \in I$.

Further a $L(\omega)$-Chandrabhan function $\beta$ is called $L^{1}(\omega)$-Chandrabhan if

(iii) for each real number $\rho>0$, there exists a function $h_{\rho}: \Omega \rightarrow L^{1}(I, \mathbb{R})$ such that

$$
|\beta(t, x, \omega)| \leq h_{\rho}(t, \omega) \text { a.e. } t \in I,
$$

for all $\omega \in \Omega$ and for all $x \in C$ with $\|x\|_{C} \leq \rho$.

Finally, a $L(\omega)$-Chandrabhan function $\beta$ is called $L_{C}^{1}(\omega)$-Chandrabhan if

(v) there exists a function $h: \Omega \rightarrow L^{1}(I, \mathbb{R})$ such that

$$
|\beta(t, x, \omega)| \leq h(t, \omega) \text { a.e. } t \in I,
$$

for all $\omega \in \Omega$ and for all $x \in C$. 
Definition 4.4. A measurable function $a: \Omega \rightarrow C(J, \mathbb{R})$ is called a lower random solution of the FRDE (1.1) if for each $\omega \in \Omega$,

$$
\begin{gathered}
\left(\frac{a(t)}{f(t, a(t, \omega), \omega)}\right)^{\prime} \leq g\left(t, a_{t}(\omega), \omega\right), \quad \text { a.e. } t \in I \\
a_{0}(\omega) \leq \phi(\omega) .
\end{gathered}
$$

Similarly, a measurable function $b: \Omega \rightarrow C(J, \mathbb{R})$ is called an upper random solution of the FRDE (1.1) if the above inequalities are satisfied with reverse signs.

We consider the following hypotheses in the sequel.

(f4) $f$ defines a function $f: J \times \mathbb{R} \times \Omega \rightarrow \mathbb{R}^{+}$with $\phi(0, \omega) \geq 0$.

(f5) For each $\omega \in \Omega$, the function $f(t, x, \omega)$ is monotone nondecreasing in $x \in C$ almost everywhere for $t \in I$.

(g4) $g$ is $L^{1}(\omega)$-Chandrabhan.

(g5) $g$ defines a function $g: J \times C \times \Omega \rightarrow \mathbb{R}^{+} \backslash\{0\}$.

(g6) The FRDE (1.1) has a lower random solution $a$ and an upper random solution $b$ with $a \leq b$.

Remark 4.1. Suppose that the hypotheses (g1), (g4) and (g5) hold and define a function $h: J \times \Omega \rightarrow \mathbb{R}$ by

$$
h(t, \omega)=\left|g\left(t, b_{t}(\omega), \omega\right)\right|=g\left(t, b_{t}(\omega), \omega\right), t \in I,
$$

for all $\omega \in \Omega$. Then the function $\omega \mapsto h(t, \omega)$ is measurable and $t \mapsto h(t, \omega)$ is Lebesgue integrable for each $\omega \in \Omega$. Further, we have for every $x \in[a, b]$,

$$
\left|g\left(t, x_{t}(\omega), \omega\right)\right| \leq h(t, \omega), \quad t \in I \text { and for all } \omega \in \Omega .
$$

Theorem 4.2. Assume that the hypotheses (f1)-(f5), (g1), (g4)-(g5) hold. Then the FRDE (1.1) has a minimal random solution $\xi_{*}$ and a maximal random solution $\xi^{*}$ in $[a, b]$, whenever

$$
\|\alpha(\omega)\|\left(\|\phi(\omega)\|_{C}+\|h(\omega)\|_{L^{1}}\right)<1,
$$

where $h(t, \omega)$ is a function as given in Remark 4.1. Moreover,

$$
\xi_{*}(\omega)=\lim _{n} x_{n}(\omega) \text { and } \xi^{*}(\omega)=\lim _{n} y_{n}(\omega),
$$

where,

$$
x_{n+1}(t, \omega)=\left\{\begin{array}{l}
{\left[f\left(t, x_{n}(t, \omega), \omega\right)\right]\left(\phi(0, \omega)+\int_{0}^{t} g\left(s, x_{n}(s+\theta, \omega), \omega\right) d s\right), \text { if } t \in I,} \\
\phi(t, \omega), \text { if } t \in I_{0}
\end{array}\right.
$$


for all $n \geq 0$ with $x_{0}(t, \omega)=a(t, \omega) \forall t \in J$; and

$$
y_{n+1}(t, \omega)=\left\{\begin{array}{l}
{\left[f\left(t, y_{n}(t, \omega), \omega\right)\right]\left(\phi(0, \omega)+\int_{0}^{t} g\left(s, y_{n}(s+\theta, \omega), \omega\right) d s\right), \text { if } t \in I,} \\
\phi(t, \omega), \text { if } t \in I_{0},
\end{array}\right.
$$

for all $n \geq 0$ with $y_{0}(t, \omega)=b(t, \omega) \forall t \in J$.

Proof. Let $X=C(J, \mathbb{R})$. Define the operators $A, B: \Omega \times X \rightarrow X$ by (3.4) and (3.5) respectively. We shall show that the operators $A(\omega)$ and $B(\omega)$ satisfy all the conditions of Theorem 4.1. It is shown, as in the proof following Theorem 3.1, that $A(\omega)$ and $B(\omega)$ are Lipschitz and completely continuous random operators respectively. Here the Lipschitz constant of $A(\omega)$ is $\|\alpha(\omega)\|$ and $M(\omega)=\|B(\omega)([a, b])\|$. By $(\mathrm{H} 8), A(\omega)$ and $B(\omega)$ are positive random operators on $X$. To prove they are monotone nondecreasing, let $x, y \in X$ be such that $x \leq y$. Then by (f5), for each $\omega \in \Omega$,

$$
\begin{aligned}
A(\omega) x(t) & =f(t, x(t, \omega), \omega) \\
& \leq f(t, y(t, \omega), \omega) \\
& =A(\omega) y(t)
\end{aligned}
$$

for all $t \in I$, and $A(\omega) x(t)=0=A(\omega) y(t)$ for all $t \in I_{0}$. Hence $A(\omega) x \leq A(\omega) y$ for each $\omega \in \Omega$. Similarly by $(\mathrm{g} 4)$,

$$
\begin{aligned}
B(\omega) x(t) & =\phi(0, \omega)+\int_{0}^{t} g\left(s, x_{s}(\omega), \omega\right) d s \\
& \leq \phi(0, \omega)+\int_{0}^{t} g\left(s, y_{s}(\omega), \omega\right) d s \\
& =B(\omega) y(t)
\end{aligned}
$$

for all $t \in I$, and $B(\omega) x(t)=\phi(t, \omega)=B(\omega) y(t)$ for all $t \in I_{0}$. Hence $B(\omega) x \leq B(\omega) y$ for each $\omega \in \Omega$. Hence $A(\omega)$ and $B(\omega)$ are monotone nondecreasing on $X$.

Next we show that condition (c) of Theorem 2.1 is satisfied. Since $a$ is lower random solution to the FRDE (1.1), we have

$$
\begin{aligned}
a(t, \omega) & \leq[f(t, a(t, \omega), \omega)]\left(\phi(0, \omega)+\int_{0}^{t} g\left(s, a_{s}(\omega), \omega\right) d s\right) \\
& =A(\omega) a(t) B(\omega) a(t)
\end{aligned}
$$

for all $t \in I$ and $\omega \in \Omega$. Similarly, $a(t, \omega) \leq \phi(t, \omega)=A(\omega) a(t) B(\omega) a(t)$ for all $t \in I_{0}$ and $\omega \in \Omega$. In consequence, $a(\omega) \leq A(\omega) a B(\omega) a$ for all $\omega \in \Omega$. Similarly, since $b$ is an upper random solution of the FRDE (1.1), it is proved that

$$
A(\omega) b B(\omega) b \leq b(\omega), \quad \forall \omega \in \Omega
$$


Now

$$
\begin{aligned}
M(\omega) & =\|B(\omega)([a, b])\| \\
& =\sup \{\|B(\omega) x\|: x \in[a, b]\} \\
& \leq\|\phi(\omega)\|_{C}+\sup \left\{\max _{t \in J}\left|\int_{0}^{t}\right| g\left(s, x_{s}(\omega), \omega\right) \mid d s: x \in[a, b]\right\} \\
& \leq\|\phi(\omega)\|_{C}+\int_{0}^{a} h(s, \omega) d s \\
& =\|\phi(\omega)\|_{C}+\|h(\omega)\|_{L^{1}}
\end{aligned}
$$

and so $\alpha(\omega) M(\omega)=\|\alpha(\omega)\|\left(\|\phi(\omega)\|_{C}+\left\|h_{q}(\omega)\right\|_{L^{1}}\right)<1$ for each $\omega \in \Omega$, where $h$ is a function as given in Remark 4.1. Hence an application of Theorem 4.1 yields that the FRDE (1.1) has a minimal random solution $\xi_{*}$ and a maximal random solution $\xi^{*}$ in $[a, b]$. Moreover,

$$
\xi_{*}(\omega)=\lim _{n} x_{n}(\omega) \text { and } \xi^{*}(\omega)=\lim _{n} y_{n}(\omega),
$$

where $\left\{x_{n+1}(\omega)\right\}$ and $\left\{x_{n+1}(\omega)\right\}$ are defined by (4.1) and (4.2) respectively.

\section{Notes and comments}

The study of deterministic nonlinear integral equations of a mixed type in a Banach algebra has been initiated in recent years by Dhage [4]. This study is motivated by Chandrasekhar's H-equation in a radiative heat transfer 2]. Therefore, some new fixed point theorems in Banach algebras, involving the product of two operators, have been developed for this purpose. The work along this line is the most active area of research and the field is growing very rapidly. See Dhage [8], Dhage and O'Regan [10] and the references therein. Here we point out that the processes of radiative heat transfer usually involve a coefficient of the heat transfer depending upon the medium of the heat conduction which would definitely play a significant role in the underlined phenomenon. This unknown coefficient may be considered as a random parameter and, in our opinion, the indeterministic form of the Chandrasekhar's H-equation is more suitable to the needs of the specialists engaged in the study of accurate behavior of the said phenomena. Hence the study of the nonlinear functional random differential and integral equations in Banach algebras has got importance. In the present paper, we have discussed a sufficiently general form of nonlinear functional random differential equations and studied only the existence theorems. However, other aspects such as stability and continuous dependence on the initial data as well as with respect to the random parameter could be discussed in a similar way. Again, the random fixed point theorems developed in the section 4.3 have some nice applications to other nonlinear random integral equations of a mixed type in a Banach algebra and some of the results in this direction will be reported elsewhere. 


\section{References}

[1] T. A. Burton and C. Kirk, A fixed point theorem of Krasnoselskii-Schaefer type, Math. Nachr. 189(1998), 23-31.

[2] S. Chandrasekhar, Radiative Heat Transfer, Dover, New York, 1980.

[3] B. C. Dhage, Monotone iterative technique for Carathéodory theory of nonlinear functional random integral equations, Tamkang J. Math. 33(2002), pp. 341-351.

[4] B. C. Dhage, On some nonlinear alternatives of Leray-Schauder type with applications to functional integral equations I, Arch. Math. (Brno) 42(2006), 11-23.

[5] B. C. Dhage, A random version of Schaefer's fixed point theorem with applications to functional random integral equations, Tamkang J. Math. 35(2004), 197-205.

[6] B. C. Dhage, A random version of a Schaefer type fixed point theorem with applications to functional random integral equations, Nonlinear Functional Analysis \& Applications $\mathbf{9}(2004), 389-403$.

[7] B. C. Dhage, A nonlinear alternative in Banach algebras with applications to functional differential equations, Nonlinear Funct. Anal. \& Appl. 9(2004), 563-575.

[8] B. C. Dhage, Existence theory for first order functional random differential equations, Nonlinear Functional Analysis \& Applications 11(2006), 227-246.

[9] B. C. Dhage, A functional integro-differential equation in Banach algebras, Functional Diff. Equations 11(3-4)(2004), 321-332.

[10] B. C. Dhage and D. O'Regan, A fixed point theorem in Banach algebras with applications to functional integral equations, Functional Diff. Equations 7(2000), 259-267.

[11] B. C. Dhage, S. N. Salunkhe, Ravi P. Agarwal and W. Zhang, A functional differential equations in Banach algebras, Math. Ineq. Appl. 8 (2005), 89-99.

[12] B. C. Dhage, J. Henderson, R. N. Ingle and S. M. Kang, Nonlinear perturbed random boundary value problems, Comm. Appl. Nonlinear Anal. 11(2004), 91-107.

[13] S. Heikkila and V. Lakshmikantham, Monotone Iterative Technique for Nonlinear Discontinuous Differential Equations, Marcel Dekker Inc., New York, 1994.

[14] S. Itoh, Random fixed point theorems with applications to random differential equations in Banach spaces, J. Math. Anal. Appl. 67(1979), 261-273.

[15] M. A. Krasnoselskii, Topological Methods in the Theory of Nonlinear Integral Equations, Cambridge University Press, London, 1964.

[16] K. Kuratowskii, and C. Ryll-Nardzewskii, A general theorem on selectors, Bull. Acad. Polon. Sci. Ser. Math. Sci. Astron. Phys. 13(1965), 397-403.

Kasubai, Gurukul Colony, Ahmedpur-413 515, Dist: Latur, Maharashtra, India.

E-mail: bcd20012001@yahoo.co.in 